\title{
Development of a two-step cultivation strategy for the production of vitamin B12 by Bacillus megaterium
}

Yousef Mohammed ${ }^{1,2}$, Byong Lee ${ }^{1,3^{*}}$, Zhen Kang ${ }^{1,2}$ and Guocheng Du ${ }^{1,2^{*}}$

\begin{abstract}
Background: Vitamin B12 is a fascinating molecule which acts as a co-factor in the metabolism of many organisms, especially affecting DNA synthesis and regulation, fatty acid synthesis and energy production. The synthesis of vitamin B12 is limited to a few of bacteria and archaea. Therefore, industrial microbial fermentation is used to meet annual demands worldwide of vitamin B12 and as an alternative method to the chemical synthesis which requires at least 60 steps that is uneconomical. Bacillus megaterium is one of vitamin B12 producers and an ideal host for many biotechnology applications and being one of the best tools for the industrial production of several enzymes. Therefore, a two-step optimization strategy was established to produce high yield of vitamin B12 by B. megaterium through the provision of the production requirements and the suitable conditions for the biosynthesis of vitamin B12.

Results: We achieved the optimum conditions for the fermentation process of $B$. megaterium to produce high yield of vitamin B12 in a practical way based on statistical design and analysis which allowed vitamin B12 production to increase up to 759 -fold $(204.46 \mu \mathrm{g} / \mathrm{l})$ as compared with control without parameters $(0.26 \mu \mathrm{g} / \mathrm{L})$. High performance liquid chromatography coupled to variable wavelength detector and mass spectrometry has been used to identify vitamin B12 forms and confirm the results.
\end{abstract}

Conclusions: We developed the fermentation process of $B$. megaterium to enhance the production of vitamin $B 12$ by providing the required supplements for the synthesis of vitamin $B 12\left(\mathrm{CoCl}_{2}, \delta\right.$-aminolevulinic acid (ALA) and 5,6-dimethylbenzimidazole (DMB)) and dividing the fermentation process into three stages. In addition, the optimum incubation times of the three fermentation stages were investigated and performed with reducing number of experimental and evaluated multiple parameters and their interactions by using statistical experimental design and analysis. All of these strategies has proven successful in enhancing the production of vitamin B12 up to $204.46 \mu \mathrm{g} / \mathrm{l}$ and demonstrated that B. megaterium could be a good candidate for the industrial production of vitamin B12.

Keywords: Vitamin B12, Bacillus megaterium, 8-Aminolevulinate (ALA), 5, 6-dimethylbenzimidazole (DMB)

\section{Background}

Vitamin B12 is one of the smallest but structurally complex molecule widely recognized in the field of biological sciences [1]. It is a water-soluble vitamin that functions as a cofactor essential in fat biochemical reactions, protein metabolism and hemoglobin synthesis as well as DNA synthesis and regulation. It comprises of two main

\footnotetext{
* Correspondence: byong.lee@jiangnan.edu.cn; gcdu@jiangnan.edu.cn ${ }^{1}$ The Key Laboratory of Industrial Biotechnology, Ministry of Education, Jiangnan University, Wuxi 214122, China

${ }^{3}$ Department of Food Science/Agricultural Chemistry, McGill University, Ste-Anne-de-Bellevue, QC H9X3V9, Canada

Full list of author information is available at the end of the article
}

coenzyme forms; methylcobalamin and adenosylcobalamin [2,3]. However, vitamin B12 commercially known as cyanocobalamin is a product formed by substituting adenosyl and methyl with a cyano group during the extraction process to ensure stability [4]. Industrial microbial fermentation is used as an alternative method to the originally established vitamin B12 chemical synthesis which requires at least 60 steps [5]. The natural process of vitamin B12 is synthesis by approximately 30 enzymemediated steps proceeding either through aerobically evident in Pseudomonas denitrificans, or anaerobically as witnessed in Bacillus megaterium, Propionibacterium shermanii, Salmonella typhimurium and Lactobacillus reuteri [6-8]. 
Bacillus megaterium, a gram-positive bacterium is one of the first established vitamin B12 producers [8] and has recently been embraced as a perfect model for anaerobic vitamin B12 biosynthesis thereby aiding in understanding the control and regulation of the cobalamin pathway as well as a biochemistry insight of the pathway [9]. Furthermore B. megaterium is a GRAS organism and the other features that make this strain an ideal host for the genetic modification and industrial production of vita$\min \mathrm{B} 12[10,11]$.

Several studies have extensively assessed the genetic and biochemical aspects regarding vitamin B12 production pathway in B. megaterium. Moreover, attempts have been carried out in other study to engineer the bacteria using metabolic engineering tools [12] but the final concentration of vitamin B12 was not high $(8 \mu \mathrm{g} / \mathrm{l})$ probably due to the lack of understanding due to the lack of understanding the pathway of vitamin B12 and production conditions of $B$. megaterium on vitamin B12.

Establishing the production requirements and understanding of the entire vitamin B12 pathway are vital in optimizing the fermentation process to increase the productivity. Although known as a strict aerobe, B. megaterium can produce vitamin B12 under a very low oxygen concentrations through a complex pathway that involves at least 25 conversion steps from $\delta$-aminolevulinate (ALA) to 5, 6-dimethylbenzimidazole (DMB) but it still requires oxygen to allow for the completion of the formation of vitamin $[13,14]$.

The aim of this research was to optimize the fermentation process of $B$. megaterium using two-step optimization strategies to produce high yields of vitamin B12. In the first strategy, the fermentation process was modified by dividing into three stages. At the first stage, the bacteria was grown aerobically to increase the biomass, followed by anaerobic growth stage for production vitamin B12 precursor and in the third stage, whole culture aeration step was used to complete vitamin B12 formation. In addition, the significance of $\mathrm{CoCl}_{2}$, ALA and DMB as supplements have been investigated. In the second strategy, the optimum time for each stage in the three stages of fermentation process was investigated and optimized using Box-Behnken design combined with Response surface methodology (RSM) to investigate the most suitable time for each stage in order to achieve high yield of vitamin B12. To our knowledge this is the first report that addresses the fermentation process of $B$. megaterium to produce vitamin B12.

\section{Results and discussion Experimental design}

A two-step optimization strategy was employed to increase the production of vitamin B12 by Bacillus megaterium through the provision of the required supplements for the synthesis of vitamin $\mathrm{B} 12(\mathrm{CoCl} 2, \delta$-aminolevulinic acid (ALA) and 5,6-dimethylbenzimidazole (DMB)) and the division of the fermentation process into three stages. In addition, the incubation times of the three fermentation stages were investigated and optimized in a practical way based on statistical design and analysis. All the measurements were done using High performance liquid chromatography and mass spectrometry to estimate vitamin B12 quantitatively and qualitatively.

\section{Confirmation of vitamin B12 analysis}

In nature, there are many of vitamin B12 analogues and some of them are not active for human and have no any economic value, especially for B. megaterium as one of the anaerobic vitamin B12 producers reveals pseudovitamin B12 (inactive form of vitamin B12) $[9,15]$. Therefore, there was a need to use HPLC and MS analysis to confirm the active forms of vitamin B12 (cyanocobalamin \& adenosylcobalamin) unlike unreliable analysis methods such as cobalamin bioassay which is not accurate and measures all of the vitamin b12 analogues without distinction, including the inactive form of vitamin B12.

During the analyzing process, UV-vis spectra data and retention times obtained by HPLC analysis showed that B. megaterium produces two major forms of vitamin B12 cyanocobalamin and adenosylcobalamin due to the transformation efficiency of the cell extraction (to replace the adenosyl and methyl group to cyano group) but the yield was not high enough. By contrary, the transformation efficiency of Propionibacterium freudenreichii cell extraction was high in our separate experiments (data not shown). Therefore, the total concentration of vitamin B12 was used as response.

The cell-extracts of $B$. megaterium revealed a peak with a retention time of 15.10 min which is agreed with the cyanocobalamin standard (15.10 min) as shown in Figure 1. The cell extraction also showed a peak with a retention time $17.007 \mathrm{~min}$ (Figure 2A), that is in agreement with the adenosylcobalamin standard (17.093 min) in Figure 2B.

The mass spectrometry data (Figure 3 ) showed that the mass spectra of the isolated compounds produced by $B$. megaterium are 1355.56 and 1579.71 that are very similar values of vitamin B12 analogues standard cyanocobalamin and adenosylcobalamin 1355.55 and 1579.65 , respectively. Consequently, these data showed the HPLC reliability and support the results. All of the data thus demonstrated the ability of $B$. megaterium to produce adenosylcobalamin which was transformed some to cyanocobalamin during the extraction process.

\section{The first step of optimization}

In this part of the experiment, we evaluated the influence of $\mathrm{CoCl}_{2}, \mathrm{ALA}$ and DMB as supplements on vitamin B12 production by $B$. megaterium. In addition, we divided the 


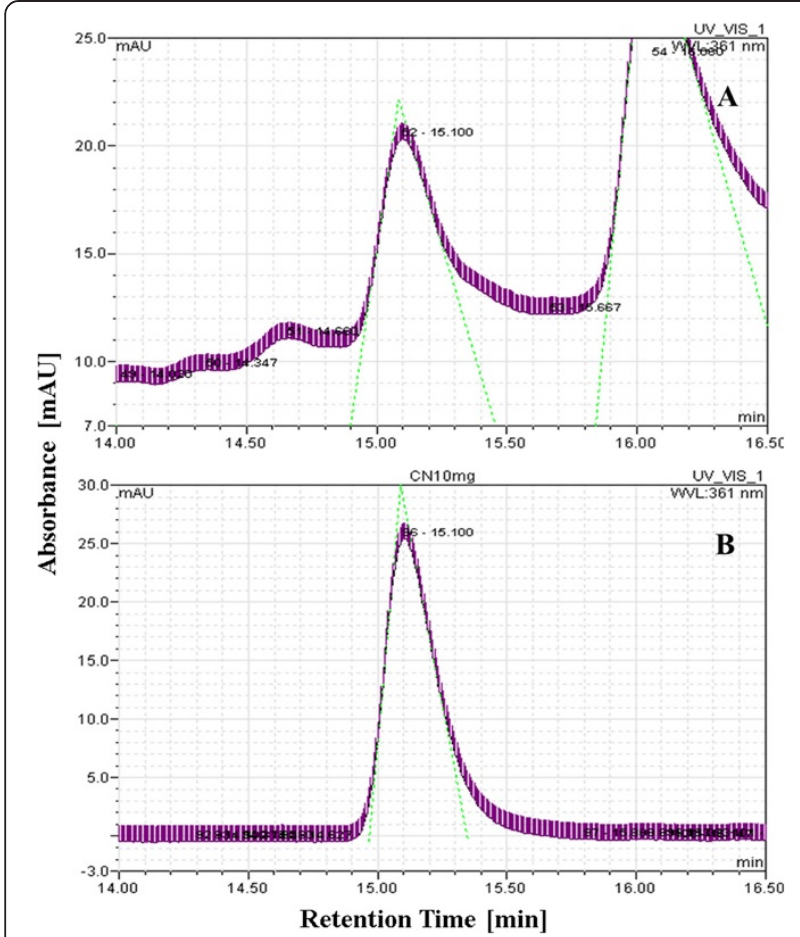

Figure 1 HPLC chromatogram of cell extraction of $B$. megaterium for sample (A) and standard cyanocobalamin (B).

fermentation process into three stages. This approach has proven successful. As shown in Figure 4, more than 170fold increase of vitamin B12 was achieved by wild type of B. megaterium $(44.38 \mu \mathrm{g} / \mathrm{l})$, as compared with control without the parameter studies $(0.26 \mu \mathrm{g} / \mathrm{l})$ in this study and others [16]. Two major forms of vitamin B12, cyanocobalamin and adenosylcobalamin were present in the cell extraction of $B$. megaterium confirmed by UV spectra and MS data.

The starting point of this step was to optimize the first part of vitamin B12 pathway (uroporphyrinogen III synthase) and consequently increase the final product (vitamin B12). $\delta$-aminolevulinic acid (ALA) is the key precursor of uroporphyrinogen III biosynthesis and at the beginning of vitamin B12 pathway [7,9]. Many studies have been focused on increasing the productivity of ALA by the overexpression of some genes (hemA, hemL) in order to enhance the production of vitamin B12 [16,17]. In our study, we added ALA (100 mg/l) directly into the culture medium and this led to more than 21-fold $(5.66 \mu \mathrm{g} / \mathrm{L}) \mathrm{im}$ provements in vitamin B12 (Figure 4). Although a large part of ALA flowed towards hemE along the branched pathway, several attempts were made to reduce the flow towards this pathway and also to reduce the possibility of harmful high hemE concentrations [16].

Regarding the final part of vitamin B12 pathway (adenosylcobinamide bioconversion), it is not completely elucidated. It required for the conversion of adenosylcobinamide to adenosylcobalamin (vitamin B12) through the replacement of the GDP group of adenosyl-GDP-cobinamide with $\alpha$-ribazole which consists of the 5, 6-dimethylbenzimidazole (DMB) attached to ribose [18]. B. megaterium has proven to produce an inactive vitamin B12 (pseudovitamin-B12) instead of vitamin B12 like most of the anaerobic producers of vitamin B $12[9,15]$ due to the failure to provide the suitable conditions for the synthesis of DMB to complete the formation of vitamin B12. In our studies two strategies were used to optimize the last part of the anaerobic biosynthesis of vitamin B12 (adenosylcobinamide bioconversion) by adding DMB (100 mg/l) at aerobic incubation in the last phase of the fermentation that resulted more than 8-fold increase in the production of vitamin B12 $(2.217 \mu \mathrm{g} / \mathrm{L})$ as indicate in Figure 4.

Finally, the fermentation process of $B$. megaterium was divided into three stages. At the first stage, the cells were grown aerobically to increase the biomass and in the second stage, the bacteria were grown anaerobically to produce vitamin B12 precursor. Subsequently, vitamin B12 formation was completed by aeration of the whole culture. Taking into account, the vitamin B12 precursors ALA and DMB were added into the fermentation medium. As shown in Figure 4 the concentration of vitamin B12 was significantly increased up to $44.38 \mu \mathrm{g} / \mathrm{l}$. Therefore, this factor was considered in the next step of our study.

\section{The second step of optimization}

The experimental runs for the Box-Behnken design are shown in Table 1. The 17 runs were used to address the effects of three factors (incubation time for three stages of the fermentation process) on three responses (biomass, total concentration of vitamin B12, amount of vitamin B12 produced per g dry cells) as shown in Additional file 1.

According to the results, the second step of the optimization led to fruitful results. In addition, the ANOVA analysis for all of the responses indicated that the models used to fit the responses variables were significant $(\mathrm{p}<0.05)$ and adequate to represent the relationship between the responses and the independent variables. Moreover, the coefficients of determination $\left(R^{2}\right)$ of the results were more than $90 \%$, which indicated that the results adequately represented the real relationship between the variables under consideration as shown in Additional files 2, 3, 4 and 5.

For the biomass, the optimal bacterial biomass was 15.8 gram dry cell weight per liter culture as shown in Figure 5 and it was obtained from sample number 10 under incubation time $9 \mathrm{~h}$ for the first stage of the fermentation, $24 \mathrm{~h}$ for the second stage and $6 \mathrm{~h}$ for the final stage. It was clear that the highest biomass yield could be achieved by shortening the incubation time for the first and third fermentation stages when the second period of the fermentation was constant at $24 \mathrm{~h}$. 


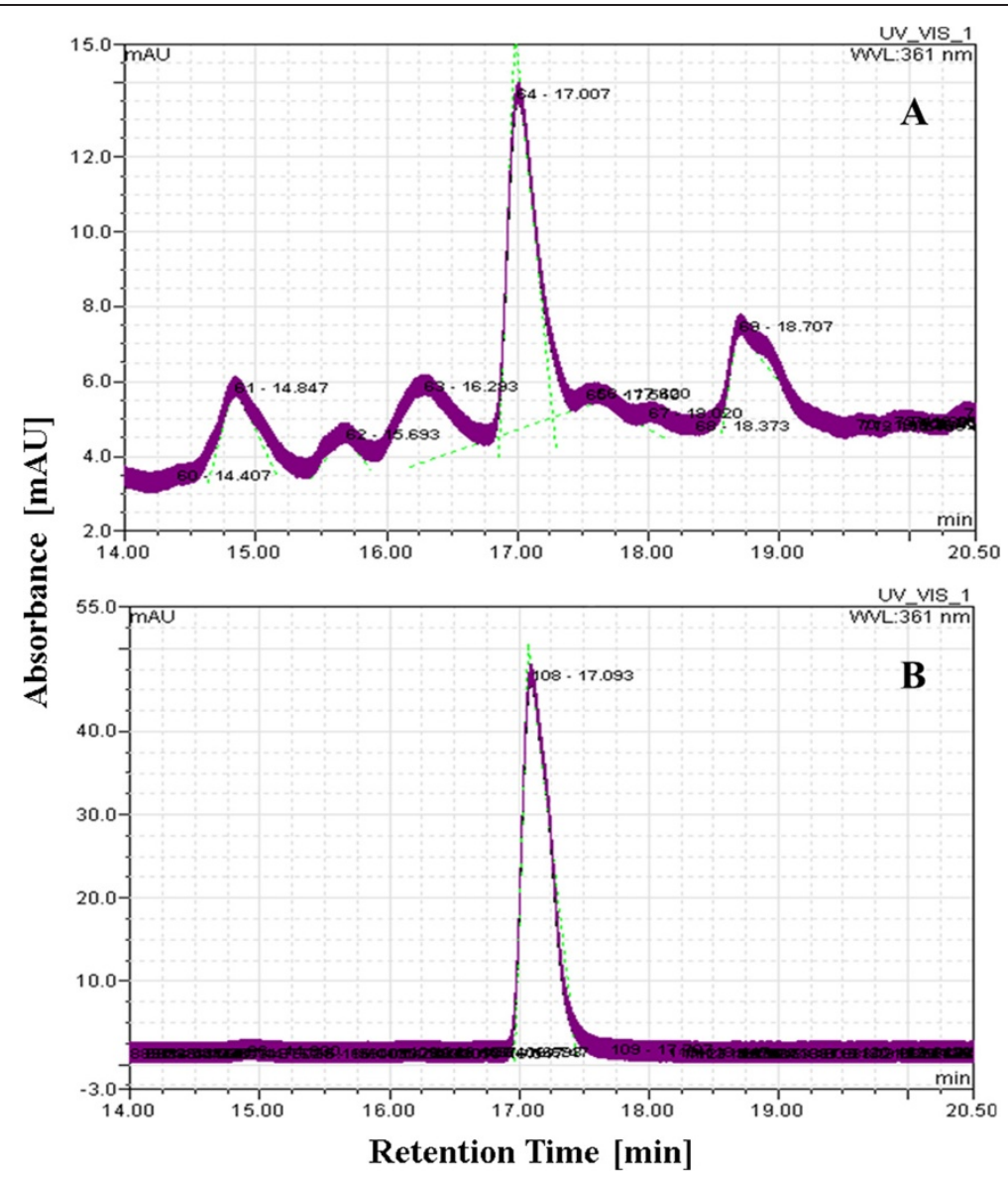

Figure 2 HPLC chromatogram of cell extraction of B. megaterium for sample (A) and standard adenosylcobalamin (B).

Furthermore, the biomass was $13.3 \mathrm{~g}$ DCW/l under the optimal conditions for the total vitamin B12 concentration. It has been noticed that the anaerobic condition has no substantial effects on the biomass even without the first aerobic stage. In this case, the logarithmic growth phase of the bacteria has lasted during all the second stage of the fermentation until the third stage. Moreover, we could overcome this issue by increasing the amount of the inoculation of the bacteria at the beginning of the fermentation process.

In the cases of the total vitamin B12, Figure 6 indicates that the highest concentration of the total vitamin B12 per liter was achieved in sample number 3 by reducing the incubation time for the first stage to $0 \mathrm{~h}$ with increasing the incubation time for the second stage up to $24 \mathrm{~h}$ during all levels of the third stage especially at $12 \mathrm{~h}$. In contrast, the increasing the incubation time for the first stage with reducing the period of the second stage led to a significant reduction in the yield of vitamin B12 as shown in Figure 6 in the sample number 8 and 11.

In relation to the final response, vitamin B12 concentrations per $\mathrm{g} D C W$, the highest concentration was in sample number 3 as shown in Figure 7. A remarkable increase in the concentration of vitamin B12 per g DCW was achieved by reducing the incubation time for the first stage and increasing the time of the second stage with incubation time approximately $12 \mathrm{~h}$ in third stage. While the lowest concentration of vitamin B12 per $\mathrm{g}$ DCW was in sample number 11 due to the increase of the incubation time for the first stage and the decrease of the incubation time for the second stage.

Both of the total vitamin B12 and the concentration of vitamin B12/g DCW have shown significant agreement and compatibility. The optimal yield of vitamin B12 observed in 17 runs of the experimental design was $204.456 \mu \mathrm{g} / \mathrm{l}$ and $15.3342 \mu \mathrm{g} / \mathrm{g}$ DCW during $24 \mathrm{~h}$ for the second stage and $12 \mathrm{~h}$ for the third stage without the first stage fermentation. In general, a significant increase in the productivity was achieved when the first stage was canceled or reduced, accompanied by the increased time of the second stage up to $24 \mathrm{~h}$ using $6-12 \mathrm{~h}$ for the third stage. This is due to the fact that the synthesis of the precursors or intermediary metabolites of vitamin B 12 in the cells requires anaerobic conditions during the logarithmic phase of growth and that was provided by 

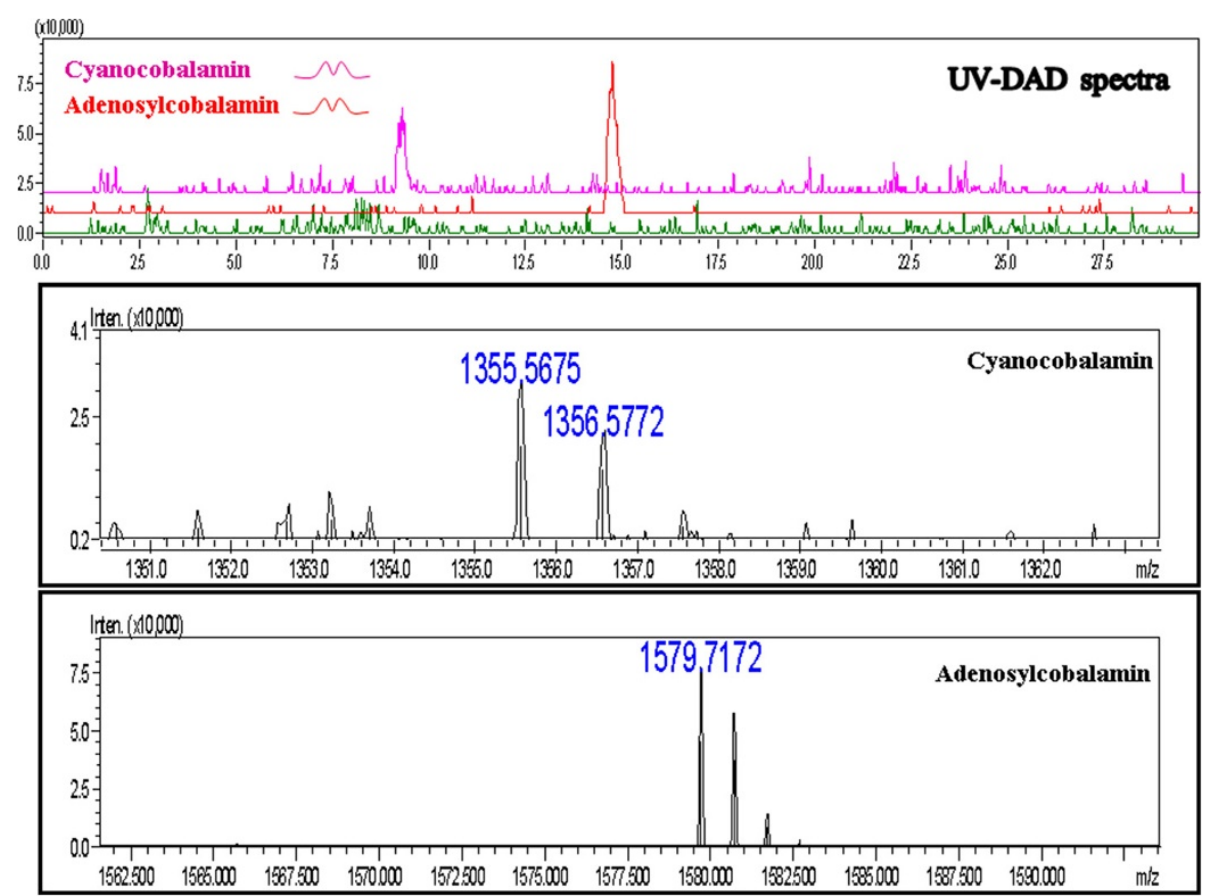

Figure 3 Mass spectrometry spectrum of the corrinoids purified from B. megaterium.

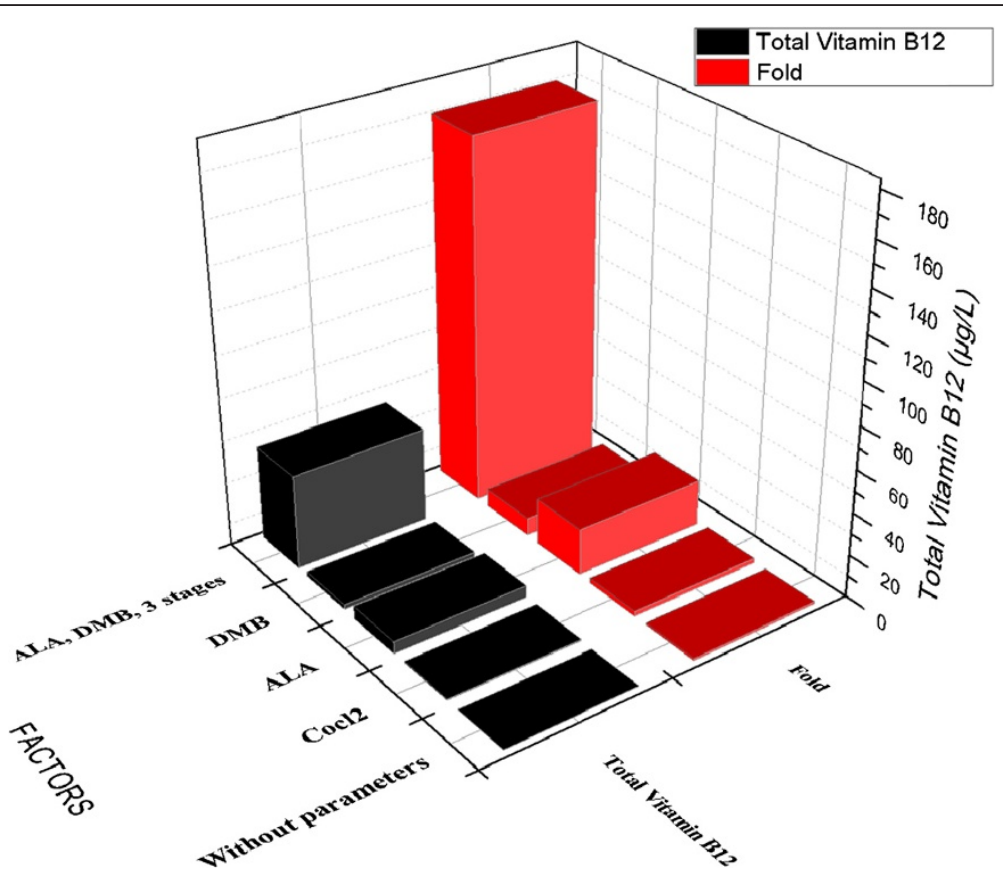

Figure 4 Total vitamin B12 content from B.megaterium under different conditions. All the measurements were done in triplicates with error margins of $10 \%$. 
Table 1 Box-Behnken matrix representing real levels of operational parameters.

\begin{tabular}{lllll}
\hline Run & $\begin{array}{l}\text { Sample } \\
\text { no. }\end{array}$ & $\begin{array}{l}\text { A: Step } \\
\mathbf{1 + O 2} \text { Hours }\end{array}$ & $\begin{array}{l}\text { B: Step } \\
\mathbf{2} \text {-02 Hours }\end{array}$ & $\begin{array}{l}\text { C: Step } \\
\mathbf{3}+\mathbf{O} 2 \text { Hours }\end{array}$ \\
\hline 1 & 8 & 18 & 15 & 18 \\
2 & 14 & 9 & 15 & 12 \\
3 & 15 & 9 & 15 & 12 \\
4 & 1 & 0 & 6 & 12 \\
5 & 6 & 18 & 15 & 6 \\
6 & 11 & 9 & 6 & 18 \\
7 & 3 & 0 & 24 & 12 \\
8 & 5 & 0 & 15 & 6 \\
9 & 17 & 9 & 15 & 12 \\
10 & 13 & 9 & 15 & 12 \\
11 & 12 & 9 & 24 & 18 \\
12 & 10 & 9 & 24 & 6 \\
13 & 4 & 18 & 24 & 12 \\
14 & 9 & 9 & 6 & 6 \\
15 & 7 & 0 & 15 & 18 \\
16 & 16 & 9 & 15 & 12 \\
17 & 2 & 18 & 6 & 12 \\
\hline & & & &
\end{tabular}

using the second stage (anaerobic conditions) directly at the beginning of the fermentation without resorting to use the first stage (aerobic conditions). During the optimum time for the third stage ( $12 \mathrm{~h})$, the biomass was significantly increased along with the completion of vitamin
B12 formation at the end of the logarithmic phase and throughout the stationary phase during the third stage of the fermentation process.

Although many studies investigated the anaerobic biosynthesis of vitamin B12 in B. megaterium in terms of genetics and biochemical characterization $[9,19]$, optimization studies of the fermentation process have not been studied. In this study, we successfully developed new strategies for the fermentation process of B. megaterium to enhance the production of vitamin B12 through the sequential fermentation methods. Two major forms of vitamin B12, cyanocobalamin and adenosylcobalamin were present in the cell extraction of B. megaterium confirmed by UV spectra and MS data. More than 750-fold increase of vitamin B12 was achieved by wild type of $B$. megaterium $(204.46 \mu \mathrm{g} / \mathrm{l})$, as compared without parameters of our study $(0.26 \mu \mathrm{g} / \mathrm{l})$ as show in Figure 8 and more than 23-fold when we compared with the modified B. megaterium $(8.52 \mu \mathrm{g} / \mathrm{l})$ from other studies $[12,16]$.

\section{Conclusions}

In this study, we directed our attention to increase the microbial biosynthesis of vitamin B12 by developing the fermentation process of B. megaterium. Two optimization steps were used to increase yield of vitamin B12 from wild type of B. megaterium. In the first step, the significance of $\mathrm{CoCl}_{2}, \mathrm{ALA}$ and DMB as supplements was investigated to increase the production of vitamin B12. In addition, we divided the fermentation process into three stages. At the

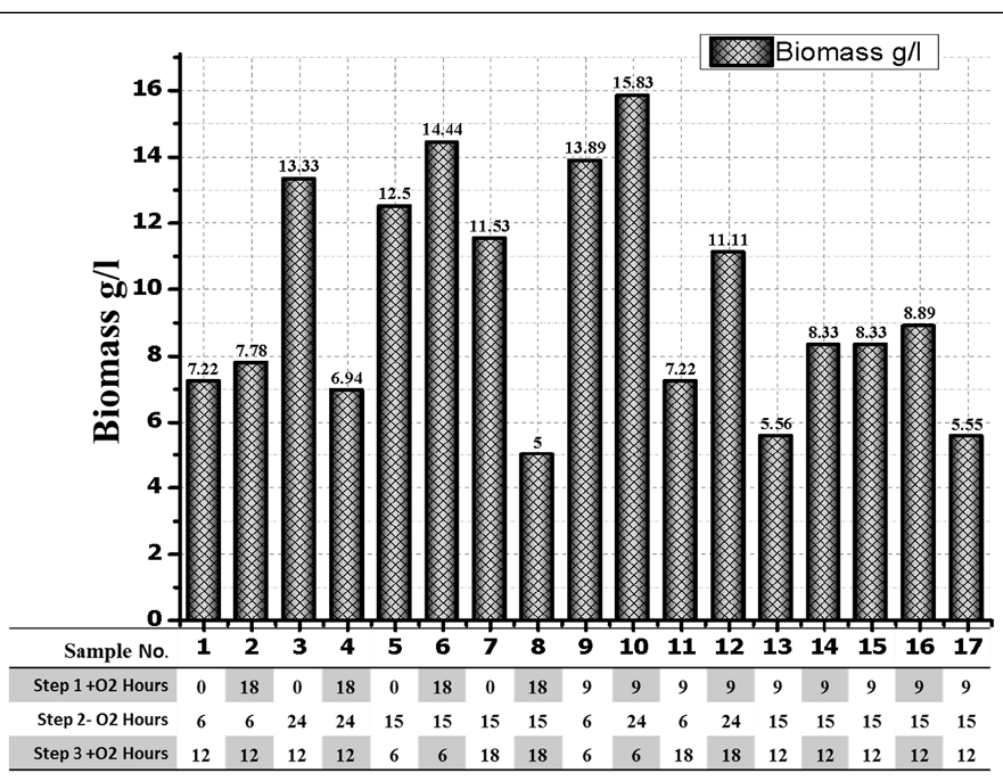

Figure 5 Biomass from the second step of optimization under different growth times in third stage of fermentation with B. megaterium. All the measurements were analyzed by ANOVA analysis with $R^{2}=94.11 \%, R^{2}$ (adjusted for $\left.d f\right)=86.54 \%, S t d$. Dev $=1.26$ and Adeq Precision $=11.310$. 


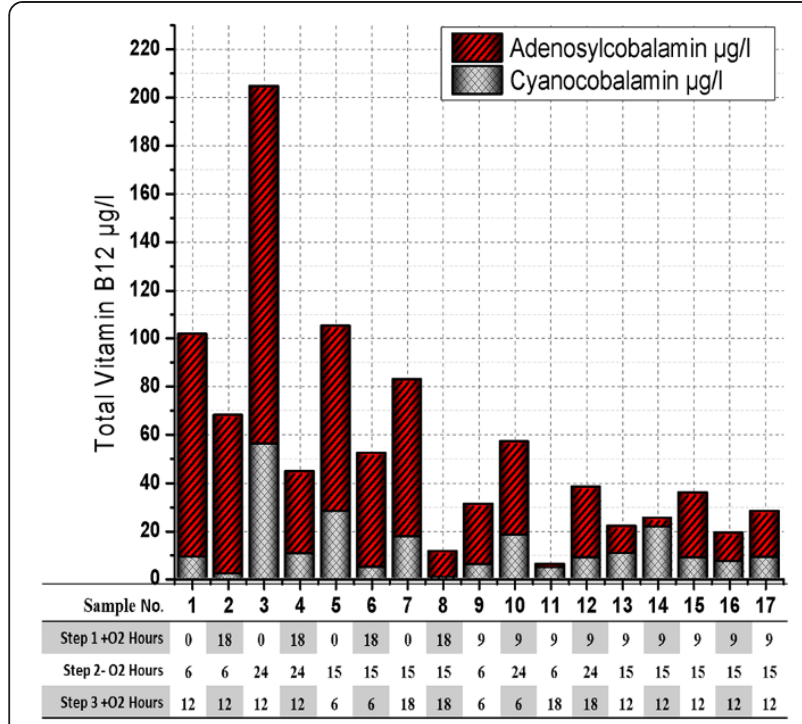

Figure 6 Total vitamin B12 content from the second step of optimization under different growth times in third stage of fermentation with B. megaterium. All vitamin B12 measurements were analyzed by ANOVA analysis with $R^{2}=97.67 \%, R^{2}$ (adjusted for $d f)=94.68 \%$, Std. Dev $=11.21$ and Adeq Precision $=22.403$. first stage, the bacteria was grown aerobically to increase the biomass, followed by anaerobic growth stage for production of vitamin B12 precursor and in the third stage, whole culture aeration step was used to complete vitamin B12 formation. Using this optimization strategy, the vitamin B12 yield was enhanced up to $44.38 \mu \mathrm{g} / \mathrm{l}$ that is an increase of more than 170-fold as compared without the parameter studies $(0.26 \mu \mathrm{g} / \mathrm{l})$. In the second strategy step, the incubation times for each stage of the three stages of fermentation were investigated and optimized using Box-Behnken design combined with Response Surface Methodology (RSM) which led to increase vitamin B12 up to 759-fold as compared with control.

From our study, we deduced the three main points to improve the production of vitamin $\mathrm{B} 12$ by B. megaterium. Firstly, the synthesis of uroporphyrinogen III which derived from $\delta$-aminolevulinic acid (ALA) could enhance ALA by some genes such as hemA and hemL or through the addition of ALA directly into the medium culture. Secondly, providing anaerobic conditions for the culture medium can enhance the synthesis of cobinamide through the oxygen-independent pathway. Thirdly, the final step of the biosynthesis of vitamin B12 includes the conversion of cobinamide to vitamin B12 through the replacement of the GDP group of adenosyl-GDP-cobinamide with $\alpha$-ribazole-p which consists of DMB attached to ribose. In our studies, we stimulated B. megaterium to complete the synthesis of vitamin B12 by adding exogenous DMB

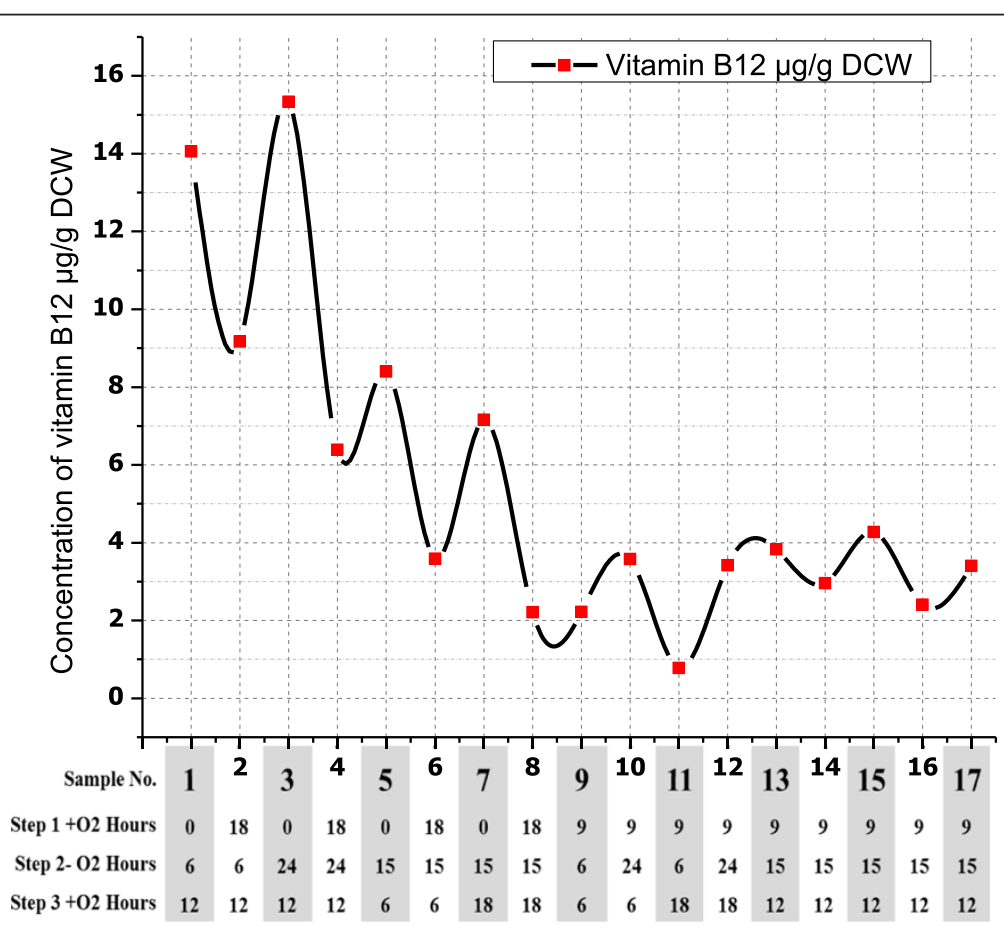

Figure 7 Vitamin B12 content/g DCW from the second step of optimization under different growth times in third stage of fermentation with B. megaterium. All vitamin B12 measurements were analyzed by ANOVA analysis with $R^{2}=97.06 \%, R^{2}$ (adjusted for $\mathrm{df}$ ) $=93.29 \%$, Std. Dev $=20.724$, mean absolute error $=5.48$ and Adeq Precision $=17.175$. 


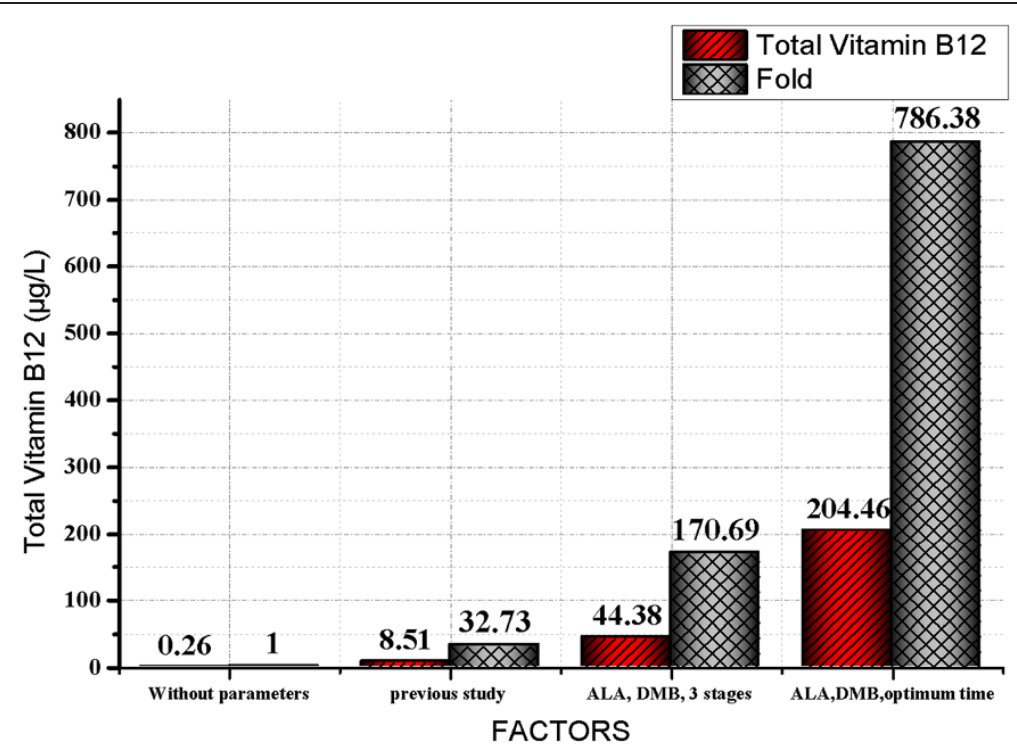

Figure 8 Total vitamin B12 content in B. megaterium during whole optimization studies.

in the culture medium under aerobic conditions in the last phase fermentation because of that bioconversion was obviously required molecular oxygen to be completed.

Further studies are underway to investigate these three points in terms of metabolic engineering through the overexpression of hemA and hemL genes form Rhodobacter sphaeroides 2.4.1 to increase ALA yield in addition to stimulate uroporphyrinogen III to flow towards cobalamin pathway instead of the hemE branched pathway by cloning cobA gene from P. freudenreichii CICC 10019. Finally, this study demonstrates that $B$. megaterium could be a good candidate for the industrial production of vitamin B12.

\section{Materials and methods Chemicals and reagents}

Cyanocobalamin, adenosylcobalamin and other chemicals were purchased from Sigma. All other chemicals were of reagent grade.

\section{Microorganisms and media}

Bacillus megaterium DSM319 wild-type strain was obtained from DSMZ (German Collection of Microorganisms and Cell Cultures, Braunschweig) and precultured in sterile Luria-Bertani (LB) medium which consisted of $10 \mathrm{~g}$ peptone, $5 \mathrm{~g}$ yeast extract, and $10 \mathrm{~g} \mathrm{NaCl}$. Modified TB medium was used for the fermentation and consisted of per liter, $30 \mathrm{~g}$ glucose, $5 \mathrm{~g}$ glycerol, $24 \mathrm{~g}$ yeast extract, and $12 \mathrm{~g}$ tryptone, $3 \mathrm{~g} \mathrm{MgSO}_{4} \cdot 7 \mathrm{H} 2 \mathrm{O}, 3 \mathrm{~g} \mathrm{CaCO}_{3}$, with $17 \mathrm{mM} \mathrm{KH}_{2} \mathrm{PO}_{4}$ and $72 \mathrm{mM} \mathrm{K} \mathrm{HPO}_{4} \cdot \mathrm{CoCl}_{2} \cdot 6 \mathrm{H}_{2} \mathrm{O}$ (100 mg/l), ALA (100 mg/l) and DMB (100 mg/l) at $\mathrm{pH} 6.8$.
Inoculum preparation and fermentation

After $2 \mathrm{ml}$ culture was inoculated into $250 \mathrm{ml}$ conical flask containing $25 \mathrm{ml}$ of LB medium and incubated for $16 \mathrm{~h}$ at $37 \mathrm{~s}$ with a speed of $200 \mathrm{rpm}, 10 \mathrm{ml}$ were transferred to the fermentation culture. The fermentation processes were carried out in batch mode during three stages. In the first stage, the bacteria were grown aerobically, followed by the anaerobic second stage, and the third stage was subjected to aerobic growth to complete the formation of vitamin B12. Aerobic cultivations were grown in $500 \mathrm{ml}$ shake flasks containing $100 \mathrm{ml}$ of modified TB at $200 \mathrm{rpm}$ and $37^{\circ} \mathrm{C}$. Anaerobic conditions were achieved by incubating in an anaerobic incubator at $37^{\circ} \mathrm{C}$. For the optimization studies, the addition of ALA, DMB and the time for each stage of the three stages of the fermentation process was dependent on the experimental design (Table 1).

\section{Preparation of cell extracts}

In order to analyze the yield of vitamin B12 obtained from B. megaterium, the bacterial cells were harvested by centrifugation $\left(6,000 \times \mathrm{g}, 10 \mathrm{~min}, 4^{\circ} \mathrm{C}\right)$, washed twice with deionized water. The bacterial cell pellet was resuspended into $1 \mathrm{~g} / 1 \mathrm{ml}$ lysozyme buffer, incubated at $37^{\circ} \mathrm{C}$ for $30 \mathrm{~min}$ and followed by ultrasonic cell disruption (Sonics \& Materials, Inc. 53 Church Hill Road, Newtown, CT 06470-1614 USA) for five times at 1-min interval under ice. The cell extraction was made up to a final volume of $20 \mathrm{ml}$ with sodium nitrite and potassium cyanide (0.5: $0.2 \mathrm{~g} / 100 \mathrm{ml}$ ) and then autoclaved at $120^{\circ} \mathrm{C}$ for $15 \mathrm{~min}$. The mixture was centrifuged $(8,000 \times \mathrm{g} ; 10 \mathrm{~min})$, 
and the supernatant was passed through the solid-phase extraction (SPE) column (500 mg C18 end-capped column with a 3-ml reservoir volume) previously activated with $2 \mathrm{ml}$ of acetonitrile. The column was washed twice with distilled water to remove salts and other hydrophilic contaminants. Subsequently, the sample was eluted with 1 volume of $50 \%$ acetonitrile and concentrated to dry in vacuum at $30^{\circ} \mathrm{C}$. The residue was dissolved in $0.5 \mathrm{ml}$ of sterile distilled water and stored in the dark at $20^{\circ} \mathrm{C}$ until further use.

\section{Design of experiments}

A two-step optimization strategy was employed to optimize vitamin B12 production by B.megaterium.

\section{The first step of optimization}

In this step, the fermentation process was modified to consist of three stages. Moreover, the addition of $\mathrm{CoCl}_{2}$, ALA and DMB as supplements were studied and compared with the control bacteria without parameters according to the previous studies. Finally, the variables were investigated in order to identify which variables have significant effects on vitamin B12 biosynthesis. Four variables $\left(\mathrm{CoCl}_{2}, \mathrm{ALA}, \mathrm{DMB}\right.$ and the division of the fermentation process into three stages) were chosen as factors. The total concentrations of vitamin B12 after the whole fermentation were used as response in this design.

\section{The second step of optimization}

In this step, a combination of RSM and Box-Behnken design optimizes the time of the three stages of fermentation which selected as the most significant variable by the last strategy step. The software package DesignExpert 8.0.6 (Stat Ease, Inc.,Minneapolis, USA) was used in this study. The factors were used at three different levels $(-1,0,+1)$ with minimum, central and maximum values. The minimum and maximum levels of each independent variable and the experimental design are shown in Table 1.

The treatment schedule for the model is given in Table 1. Five replicates (run) at the center of the design were used to estimate the pure error and sum of squares. Seventeen experiments were randomly run as shown in Table 1 . The responses obtained from the experimental design were biomass and the total vitamin B12 concentration (cyanocobalamin and adenosylcobalamin). In addition, the amount of vitamin B12 was obtained from $1 \mathrm{~g}$ of dry cells as the third response.

\section{Vitamin B12 analysis using HPLC}

The corrinoids were purified from B. megaterium cellextracts and analyzed using Dionex Ultimate 3000
(Thermo Scientific, Waltham, MA, USA) that comprised of an auto sampler, and an Ultimate 3000 Variable Wavelength detector (UV) set at $361 \mathrm{~nm}$. Vitamin B12 concentration was analyzed at a flow rate of $0.7 \mathrm{ml} / \mathrm{min}$. The system was completed with an Amethyst column type Acclaim C18-H $(5 \mu \mathrm{m}, 4.6 \times 250 \mathrm{~mm})$. The compositions of two mobile phases (A, B) were methanol: distilled water (60:40) and (10:90) respectively with added $0.07 \mathrm{~mL}$ phosphoric acid. The samples and standards were dissolved in the mobile phase and $10 \mu \mathrm{l}$ sample volume was injected into the column at $30^{\circ} \mathrm{C}$.

\section{Identification of Vitamin B12 by mass spectrometry}

Mass spectrometric analysis of $B$. megaterium cell extraction was performed by the liquid chromatography/ mass spectrometry-ion trap-time of flight (LC-MS-ITTOF) (Shimadzu, Kyoto, Japan). Analysis parameters: Electrospray ion source; Interface voltage of $-3.50 \mathrm{kV}$, Nebulizer gas flow of $1.5 \mathrm{~L}$ min -1 , CDL temperature of $200^{\circ} \mathrm{C}$, Block heater temperature of $200^{\circ} \mathrm{C}$, Detector voltage of $1.60 \mathrm{kV}$, Drying gas of $100 \mathrm{kpa}$, Ion accumulation time of $10 \mathrm{msec}$, MS range of $\mathrm{m} / \mathrm{z} 300$ to 1500 , CID parameters as energy of $50 \%$, collision gas of $100 \%$, repeated of 10 . MS data were processed with LCMS solution ver. 3.4 software (Shimadzu). Ten $\mu$ l of the sample solution was introduced into the ESI ion source of mass spectrometer via the auto sampler of HPLC system with C18 column $(150 \times 2.0 \mathrm{~mm}$; Shimadzu, Kyoto, Japan $)$.

\section{Cell dry weight}

Dry weight of biomass was estimated after centrifugation and drying to constant weight at $103-105^{\circ} \mathrm{C}$ using a vacuum oven.

\section{Statistical analysis}

The single factor experiments were analyzed using calculated means and standard deviations by using the software Origin 8.5.1.

Analysis of variance (ANOVA) was carried out by using the software package Design-Expert 8.0.6 (Stat Ease, Inc., Minneapolis, USA) and the significance of the difference between means was determined by Duncan's Multiple Range test $(\mathrm{P}<0.05)$. Values expressed are means \pm standard deviation.

\section{Abbreviations}

ALA: $\delta$-Aminolevulinate; DMB: 5,6-dimethylbenzimidazole; RSM: Response surface methodology; R2: Coefficients of determination; DCW: Dry cell weight.

\section{Competing interests}

The authors declare none of competing interests.

\section{Authors' contributions}

YM, BL, ZK and GU participated in the experimental design and established the analytical method of yield measurement. YM operated RSM software and carried out the fermentation. YM and BL participated in the statistical analysis. $B L, Z K$ and $G U$ carried out the validation and verification experiments. YM, BL, 
ZK and GU were responsible for the methods comparison and conceived of the study and helped to draft the manuscript. All authors read and approved the final manuscript.

\section{Acknowledgements}

This work was financially supported by the Major State Basic Research Development Program of China (973 Program, 2014CB745103, 2013CB733902), the National Natural Science Foundation of China (31200020), the Program for Changjiang Scholars and Innovative Research Team in University (No. IRT1135), the National Science Foundation for Post-doctoral Scientists in China (2013 M540414), the Jiangsu Planned Projects for Post-doctoral Research Funds (1301010B).

\section{Author details}

'The Key Laboratory of Industrial Biotechnology, Ministry of Education, Jiangnan University, Wuxi 214122, China. ${ }^{2}$ School of Biotechnology, Jiangnan University, Wuxi 214122, China. ${ }^{3}$ Department of Food Science/Agricultural Chemistry, McGill University, Ste-Anne-de-Bellevue, QC H9X3V9, Canada.

Received: 8 May 2014 Accepted: 6 July 2014

Published: 15 July 2014

\section{Additional files}

Additional file 1: HPLC data for Bacillus megaterium samples (the second strategy for the optimization).Additional file 2: Response surface methodology and explanation data for the second step of optimization fermentation.Additional file 3: ANOVA of Biomass.Additional file 4: ANOVA of Total vitamin B12.Additional file 5: ANOVA of Vitamins B12 per g CDW.

References

1. Warren MJ, Raux E, Schubert HL, Escalante-Semerena JC: The biosynthesis of adenosylcobalamin (vitamin B12). Nat Prod Rep 2002, 19:390-412.

2. Drennan $\mathrm{CL}$, Huang S, Drummond JT, Matthews RG, Lidwig ML: How a protein binds B12: a $3.0 \AA$ X-ray structure of B12-binding domains of methionine synthase. Science 1994, 266:1669-1674.

3. Mancia F, Keep NH, Nakagawa A, Leadlay PF, McSweeney S, Rasmussen B, Secke PB, Diat O, Evans PR: How coenzyme B12 radicals are generated: the crystal structure of methylmalonyl-coenzyme $A$ mutase at $2 \AA$ resolution. Structure 1996, 4:339-350.

4. Raux E, Schubert HL, Warren MJ: Biosynthesis of cobalamin (vitamin B12): a bacterial conundrum. Cell Mol Life Sci 2000, 57:1880-1893.

5. Martens J-H, Barg H, Warren MJ, Jahn D: Microbial production of vitamin B12. Appl Microbiol Biotechnol 2002, 58:275-285.

6. Taranto MP, Vera JL, Hugenholtz J, Valdez GFD, Sesma F: Lactobacillus reuteri CRL 1098 produces cobalamin. J Bacteriol 2003, 185:5643-5647.

7. Kang Z, Zhang J, Zhou J, Qi Q, Du G, Chen J: Recent advances in microbial production of $\delta$-aminolevulinic acid and vitamin B12. Biotechnol Adv 2012, 30:1533-1542.

8. Raux E, Lanois A, Warren MJ, Rambach A, Thermes C: Cobalamin (vitamin B12) biosynthesis: identification and characterization of a Bacillus megaterium cobloperon. Biochem J 1998, 335:159-166.

9. Moore SJ, Lawrence AD, Biedendieck R, Deery E, Frank S, Howard MJ, Rigby SEJ, Warren MJ: Elucidation of the anaerobic pathway for the corrin component of cobalamin (vitamin B12). Proc Natl Acad Sci U S A 2013, 110:14906-14911.

10. Barg H, Malten M, Jahn M, Jahn D: Protein and Vitamin Production in Bacillus Megaterium. In Microbial Processes and Products, Methods in Biotechnology, Volume 18. Edited by Barredo JL. 2005:205-223.

11. Vary PS, Biedendieck R, Fuerch T, Meinhardt F, Rohde M, Deckwer WD, Jahn $D$ : Bacillus megaterium from simple soil bacterium to industrial protein production host. Appl Microbiol Biotechnol 2007, 76:957-967.

12. Barg H, Jahn D: Method for the Production of Vitamin B12. US Patent 20060105432 A1; 2006

13. Taga ME, Larsen NA, Howard-Jones AR, Walsh CT, Walker GC: BluB cannibalizes flavin to form the lower ligand of vitamin B12. Nature 2007,

446(7134):449-453.
14. Fowler CC, Brown ED, Li Y: Using a riboswitch sensor to examine coenzyme B12 metabolism and transport in E. coli. Chem Biol 2010, 17:756-765.

15. Mohammed Y, Lee B, Kang Z, Du G: Capability of Lactobacillus reuteri to produce an active form of vitamin B12 under optimized fermentation conditions. JAIR 2014, 2:2278-5213.

16. Biedendieck R, Malten M, Barg H, Bunk B, Martens J-H, Deery E, Leech H, Warren MJ, Jahn D: Metabolic engineering of cobalamin (vitamin B12) production in Bacillus megaterium. Microbial Biotechnol 2010, 3:24-37.

17. Murooka Y, Piao Y, Kiatpapan P, Yamashita M: Production of tetrapyrrole compounds and vitamin B12 using genetically engineering of Propionibacterium freudenreichii. An overview. Dairy Sci Technol 2005 85:9-22.

18. Scott Al, Roessner CA: Recent discoveries in the pathways to cobalamin (coenzyme B12) achieved through chemistry and biology. Pure Appl Chem 2007, 79:2179-2188.

19. Moore SJ, Warren MJ: The anaerobic biosynthesis of vitamin B12. Biochem Soc Trans 2012, 40:581-586.

doi:10.1186/s12934-014-0102-7

Cite this article as: Mohammed et al:: Development of a two-step cultivation strategy for the production of vitamin B12 by Bacillus megaterium. Microbial Cell Factories 2014 13:102

\section{Submit your next manuscript to BioMed Central and take full advantage of:}

- Convenient online submission

- Thorough peer review

- No space constraints or color figure charges

- Immediate publication on acceptance

- Inclusion in PubMed, CAS, Scopus and Google Scholar

- Research which is freely available for redistribution 